PROCEEDINGS OF THE

AMERICAN MATHEMATICAL SOCIETY

Volume 117, Number 3, March 1993

\title{
FINITE SUBSTRUCTURE LATTICES OF MODELS OF PEANO ARITHMETIC
}

\author{
JAMES H. SCHMERL
}

(Communicated by Andreas R. Blass)

\begin{abstract}
Some new finite lattices (for example, $M_{4}, M_{7}$, and the hexagon lattice) are shown to be isomorphic to the lattice of elementary substructures of a model of Peano Arithmetic.
\end{abstract}

The set of elementary substructures of a model $\mathcal{N}$ of Peano Arithmetic forms a lattice $\operatorname{Lt}(\mathcal{N})$, the substructure lattice of $\mathscr{N}$. It is unknown whether there are finite lattices that are not isomorphic to any substructure lattice. Indeed, it was conjectured in [8] that for any finite lattice $L$ there is some $\mathscr{N} \vDash P A$ for which $L \cong \operatorname{Lt}(\mathscr{N})$. It was proved in [8] that if $2 \leq n<\omega$, then there is $\mathscr{N}$ such that $\operatorname{Lt}(\mathscr{N}) \cong \Pi(n)$. (The partition lattices $\Pi(n)$ are defined below.) For $3 \leq n<\omega$, the lattice $M_{n}$ is the unique lattice that has $n+2$ elements and $n$ atoms. Thus, the lattice $M_{3}$, which is isomorphic to $\Pi(3)$, is isomorphic to a substructure lattice, although it is known from [2] or [4] that if $\mathscr{N}$ is a model of True Arithmetic and $3 \leq n<\omega$, then $\operatorname{Lt}(\mathscr{N}) \not M_{n}$. Other examples of finite lattices that are not substructure lattices of models of True Arithmetic are given in [8]. The lattices $M_{n}$ for $4 \leq n<\omega$ and the hexagon lattice were identified in [8] as specific finite lattices that were not known to be isomorphic to substructure lattices. It is a consequence of the theorem in this note that $M_{n}$, whenever $n=p^{a}+1$ for some prime $p$, the hexagon lattice, and the lattice $M_{7}$ are isomorphic to substructure lattices. It is still unknown whether or not $M_{11}$ is isomorphic to a substructure lattice.

All previously known positive results about finite substructure lattices are contained in [8]. Prior to [8], Paris [4] proved that all finite distributive lattices occur as substructure lattices. (Also see [7].) Wilkie [9] showed that the pentagon lattice is a substructure lattice, and Paris [5] proved that $M_{3}$ is a sublattice of an infinite substructure lattice.

More generally, intermediate structure lattices will be considered. For $\mathscr{M} \prec$ $\mathscr{N} \vDash P A$, let $\operatorname{Lt}(\mathscr{N} / \mathscr{M})=\left\{\mathscr{M}_{0} \in \operatorname{Lt}(\mathscr{N}): \mathscr{M} \preceq \mathscr{M}_{0} \preceq \mathscr{N}\right\}$, regarded as a sublattice of $\operatorname{Lt}(\mathscr{N})$. Notice that if $\mathscr{M}$ is a minimal model of $P A$ and $\mathscr{M} \prec$ $\mathscr{N}$, then $\operatorname{Lt}(\mathscr{N})=\operatorname{Lt}(\mathscr{N} / \mathscr{M})$.

Given a set $A$, let $\Pi(A)$ be the set of all partitions of $A$. If $a, b \in A$ and $\pi \in \Pi(A)$, then we write $a \approx b(\bmod \pi)$ if $\{a, b\} \subseteq C$ for some $C \in \pi$.

Received by the editors February 11, 1991 and, in revised form, July 2, 1991.

1991 Mathematics Subject Classification. Primary 03H15, 03C62; Secondary 06B15. 
There are two extreme partitions $\mathbf{0}_{A}$ and $\mathbf{1}_{A}$ in $\Pi(A)$, where

$$
\begin{array}{ll}
a \approx b\left(\bmod \mathbf{1}_{A}\right) & \text { iff } a=b, \\
a \approx b\left(\bmod \mathbf{0}_{A}\right) & \text { for every } a, b \in A .
\end{array}
$$

We partially order $\Pi(A)$ so that if $\pi_{1}, \pi_{2} \in \Pi$ then $\pi_{1} \leq \pi_{2}$ iff $\pi_{2}$ is a refinement of $\pi_{1}$. In particular, $\mathbf{0}_{A} \leq \pi \leq \mathbf{1}_{A}$ for each $\pi \in \Pi(A)$. With this partial order, $\Pi(A)$ becomes a complete lattice (which is the dual of the lattice usually considered). Thus, if $\pi_{1}, \pi_{2} \in \Pi(A)$ then $\pi_{1} \vee \pi_{2}=\left\{C_{0}: C_{0}=\right.$ $C_{1} \cap C_{2} \neq \varnothing$ for some $C_{1} \in \pi_{1}$ and $\left.C_{2} \in \pi_{2}\right\}$.

Let $(A, \mathscr{F})$ be an algebra (in the sense of universal algebra); that is, each $f \in \mathscr{F}$ is a function $f: A^{k} \rightarrow A$ for some $k<\omega$. We let $\Pi(A, \mathscr{F})$ be the sublattice of $\Pi(A)$ consisting of all partitions of $A$ that correspond to congruences of $(A, \mathscr{F})$. Thus, $\pi \in \Pi(A, \mathscr{F})$ iff whenever $f \in \mathscr{F}$ is $k$-ary and $a_{0}, a_{1}, \ldots, a_{k-1}, b_{0}, b_{1}, \ldots, b_{k-1} \in A$ are such that $a_{i} \approx b_{i}(\bmod \pi)$ for each $i<k$, then

$$
f\left(a_{0}, a_{1}, \ldots, a_{k-1}\right) \approx f\left(b_{0}, b_{1}, \ldots, b_{k-1}\right) \quad(\bmod \pi) .
$$

The theorem can now be stated.

Theorem. Let $\mathscr{M} \vDash P A$ be countable and nonstandard, and let $(A, \mathscr{F})$ be a finite algebra. Then $\mathscr{M}$ has a cofinal elementary extension $\mathscr{N}$ such that $\operatorname{Lt}(\mathscr{N} / \mathscr{M}) \cong \Pi(A, \mathscr{F})$.

We need some definitions taken from [8].

Let $L=(L, \wedge, \vee)$ be a finite lattice. We denote the minimum and maximum elements of $L$ by 0 and 1 . A representation $\alpha$ of $L$ in $\Pi(A)$ is an injection $\alpha: L \rightarrow \Pi(A)$ such that $\alpha(0)=\mathbf{0}_{A}, \alpha(1)=\mathbf{1}_{A}$ and $\alpha(x \vee y)=\alpha(x) \vee \alpha(y)$ for each $x, y \in L$. Notice that we do not require that a representation satisfy the identity $\alpha(x \wedge y)=\alpha(x) \wedge \alpha(y)$.

Let $\alpha: L \rightarrow \Pi(A)$ be a representation. Then $\alpha$ is finite if $A$ is finite, and $\alpha$ is nontrivial if $|\alpha(x)| \neq 2$ whenever $x \in L$. The representation $\alpha: L \rightarrow$ $\Pi(A)$ is a congruence representation if there is an algebra $(A, \mathscr{F})$ such that $\alpha: L \rightarrow \Pi(A, \mathscr{F})$ is a lattice isomorphism. For $n \geq 1$ let $\alpha^{n}: L \rightarrow \Pi\left(A^{n}\right)$ be the representation such that for each $c, d \in A^{n}$ and $x \in L$,

$$
c \approx d\left(\bmod \alpha^{n}(x)\right) \Leftrightarrow \text { for every } i<n, c_{i} \approx d_{i}(\bmod \alpha(x)) .
$$

If we let $\mathscr{F}_{1}$ be the set of unary polynomial functions of $(A, \mathscr{F})$, then $\Pi\left(A, \mathscr{F}_{1}\right)$ $=\Pi(A, \mathscr{F})$. Therefore, when considering some $\Pi(A, \mathscr{F})$, we can assume that $\mathscr{F}$ consists only of unary functions.

It is known [3] that every finite lattice has a congruence representation. However, it is an open question of some apparent difficulty whether every finite lattice has a finite congruence representation. The lattice $M_{11}$ is a specific lattice that has no known finite congruence representation. We present some examples of lattices that have finite congruence representations.

Let $L$ be a finite distributive lattice. Let $A=\{x \in L: x=1$ or $x$ is a meetirreducible $\}$, considered as a subposet of $L$. Let $\mathscr{F}$ be the set of all functions $f: A \rightarrow A$ such that $f(a) \geq a$ for each $a \in A$. Then $L$ and $\Pi(A, \mathscr{F})$ are isomorphic lattices. In fact, let $\alpha: L \rightarrow \Pi(A)$ be such that for each $x \in L, \alpha(x)$ is the partition whose only equivalence class that (possibly) is not a singleton is $\{a \in A: a \geq x\}$. Then $\alpha: L \rightarrow \Pi(A, \mathscr{F})$ is a lattice isomorphism. 
Let $F$ be a finite field of order $q$, and let $n=q+1$. Let $A=F \times F$, and let $f \in \mathscr{F}$ iff there are $a, b, c, d \in F$ such that $f(x, y)=(a x+b, c y+d)$. Then $\Pi(A, \mathscr{F}) \cong M_{n}$.

An observation due to Feit [1] implies that $M_{7}$ has a finite congruence representation.

Finally, let $A=\{0,1,2,3,4,5,6\}$. Then there is $\mathscr{F}$ such that $\Pi(A, \mathscr{F})$ is isomorphic to the hexagon lattice. Specifically, $\Pi(A, \mathscr{F})=\left\{\mathbf{0}_{A}, \mathbf{1}_{A},\{\{0\}\right.$, $\{1,2\},\{3,4\},\{5,6\}\},\{\{0,1,2\},\{3,4\},\{5,6\}\},\{\{0\},\{1,3\},\{4,6\}$, $\{2,5\}\},\{\{0,4,6\},\{1,3\},\{2,5\}\}\}$.

We now proceed with the proof of the theorem.

Lemma 1. Suppose $L$ is a finite lattice, $\alpha: L \rightarrow \Pi(A)$ is a representation, and $2 \leq n<\omega$. Then

(1) $\alpha^{n}$ is a nontrivial representation of $L$;

(2) if $\alpha$ is a finite representation then so is $\alpha^{n}$;

(3) if $\alpha$ is a congruence representation then so is $\alpha^{n}$.

Proof. (1) Clearly, if $x \in L$ then $\left|\alpha^{n}(x)\right|=|\alpha(x)|^{n} \neq 2$.

(2) If $A$ is finite then so is $A^{n}$.

(3) Let $(A, \mathscr{F})$ be a unary algebra such that $\alpha: L \rightarrow \Pi(A, \mathscr{F})$ is a lattice isomorphism. Without loss of generality assume that $\mathscr{F}$ has the identity function and all constant functions as members. Let $\mathscr{G}_{0}$ be the set of all functions $g: A^{n} \rightarrow A^{n}$ for which there are $f_{0}, f_{1}, \ldots, f_{n-1} \in \mathscr{F}$ such that $g\left(\left\langle a_{0}, a_{1}, \ldots, a_{n-1}\right\rangle\right)=\left\langle f_{0}\left(a_{0}\right), f_{1}\left(a_{1}\right), \ldots, f_{n-1}\left(a_{n-1}\right)\right\rangle$. We introduce the following notation to be used for the rest of this proof: for any $x$ we denote by $\bar{x}$ the $n$-tuple $\langle x, x, \ldots, x\rangle$. Now let $\mathscr{G}_{1}$ be the set of all functions $g: A^{n} \rightarrow A^{n}$ where for some $i<n, g\left(\left\langle a_{0}, a_{1}, \ldots, a_{n-1}\right\rangle\right)=\overline{a_{1}}$. Let $\mathscr{G}=\mathscr{G}_{0} \cup \mathscr{G}_{1}$. We claim that $\alpha^{n}: L \rightarrow \Pi\left(A^{n}, \mathscr{G}\right)$ is a lattice isomorphism.

First we show that $\alpha^{n}(x) \in \Pi\left(A^{n}, \mathscr{G}\right)$ for $x \in L$. Suppose $g \in \mathscr{G}_{0}$, so that $g\left(\left\langle a_{0}, a_{1}, \ldots, a_{n-1}\right\rangle\right)=\left\langle f_{0}\left(a_{0}\right), f_{1}\left(a_{1}\right), \ldots, f_{n-1}\left(a_{n-1}\right)\right\rangle$. Then

$$
\begin{aligned}
c \approx d\left(\bmod \alpha^{n}(x)\right) & \Rightarrow \text { for all } i<n, c_{i} \approx d_{i}(\bmod \alpha(x)) \\
& \Rightarrow \text { for all } i<n, f_{i}\left(c_{i}\right) \approx f_{i}\left(d_{i}\right) \quad(\bmod \alpha(x)) \\
& \Rightarrow\left\langle f_{0}\left(c_{0}\right), \ldots, f_{n-1}\left(c_{n-1}\right)\right\rangle \\
& \approx\left\langle f_{0}\left(d_{0}\right), \ldots, f_{n-1}\left(d_{n-1}\right)\right\rangle\left(\bmod \alpha^{n}(x)\right) \\
& \Rightarrow g(c) \approx g(d)\left(\bmod \alpha^{n}(x)\right) .
\end{aligned}
$$

Next suppose $g \in \mathscr{G}_{1}$, so that $g\left(\left\langle a_{0}, a_{1}, \ldots, a_{n-1}\right\rangle\right)=\overline{a_{i}}$. Then

$$
\begin{aligned}
c \approx d\left(\bmod \alpha^{n}(x)\right) & \Rightarrow c_{i} \approx d_{i}(\bmod \alpha(x)) \\
& \Rightarrow \overline{c_{i}} \approx \overline{d_{i}}\left(\bmod \alpha^{n}(x)\right) \\
& \Rightarrow g(c) \approx g(d)\left(\bmod \alpha^{n}(x)\right) .
\end{aligned}
$$

Therefore, for all $g \in \mathscr{G}$, if $c \approx d\left(\bmod \alpha^{n}(x)\right)$ then $g(c) \approx g(d)(\bmod$ $\left.\alpha^{n}(x)\right)$. Hence, $\alpha^{n}(x) \in \Pi\left(A^{n}, \mathscr{G}\right)$.

Secondly, we show that if $\pi \in \Pi\left(A^{n}, \mathscr{G}\right)$ then $\pi=\alpha^{n}(x)$ for some $x \in L$. Let $\pi \in \Pi\left(A^{n}, \mathscr{G}\right)$, and let $\rho \in \Pi(A)$ be such that

$$
a \approx b \quad(\bmod \rho) \Leftrightarrow \bar{a} \approx \bar{b} \quad(\bmod \pi) .
$$


To show that $\rho \in \Pi(A, \mathscr{F})$, consider arbitrary $f \in \mathscr{F}$. Then

$$
\begin{aligned}
a \approx b(\bmod \rho) & \Rightarrow \bar{a} \approx \bar{b}(\bmod \pi) \\
& \Rightarrow \overline{f(a)} \approx \overline{f(b)}(\bmod \pi) \\
& \Rightarrow f(a) \approx f(b)(\bmod \rho) .
\end{aligned}
$$

Thus, $\rho \in \Pi(A, \mathscr{F})$ so that $\rho=\alpha(x)$ for some $x \in L$.

We claim that $\alpha^{n}(x)=\pi$. To see that $\alpha^{n}(x) \leq \pi$, notice that

$$
\begin{aligned}
c \approx d(\bmod \pi) & \Rightarrow \text { for each } i<n, \overline{c_{1}} \approx \overline{d_{i}}(\bmod \pi) \\
& \Rightarrow \text { for each } i<n, c_{i} \approx d_{i}(\bmod \alpha(x)) \\
& \Rightarrow c \approx d\left(\bmod \alpha^{n}(x)\right) .
\end{aligned}
$$

To show that $\pi \leq \alpha^{n}(x)$, consider $c, d \in A^{n}$ for which $c \approx d \quad\left(\bmod \alpha^{n}(x)\right)$. Let $g_{0}, g_{1}, \ldots, g_{n-1} \in \mathscr{G}_{0}$ be such that for $i<n, g_{i}\left(\left\langle a_{0}, a_{1}, \ldots, a_{n-1}\right\rangle\right)=$ $\left\langle b_{0}, b_{1}, \ldots, b_{n-1}\right\rangle$, where

$$
b_{j}= \begin{cases}d_{j} & \text { if } j<i \\ a_{j} & \text { if } j=i \\ c_{j} & \text { if } j>i\end{cases}
$$

for all $\left\langle a_{0}, a_{1}, \ldots, a_{n-1}\right\rangle \in A^{n}$. Then $c=g_{0}\left(\overline{c_{0}}\right), g_{i}\left(\overline{c_{i}}\right) \approx g_{i}\left(\overline{d_{i}}\right)(\bmod \pi)$, $g_{i}\left(\overline{d_{1}}\right)=g_{i+1}\left(\overline{c_{i+1}}\right)$, and $g_{n-1}\left(\overline{d_{n-1}}\right)=d$. Therefore, $c \approx d(\bmod \pi)$.

Definition. Let $\alpha: L \rightarrow \Pi(A)$ and $\beta: L \rightarrow \Pi(B)$ be representations of $L$. Then $\alpha$ arrows $\beta$ (in symbols: $\alpha \rightarrow \beta$ ) if whenever $\pi \in \Pi(A)$ there is an injection $\theta: B \rightarrow A$ such that

(1) whenever $x \in L$ and $a, b \in B$, then

$$
a \approx b \quad(\bmod \beta(x)) \Leftrightarrow \theta(a) \approx \theta(b) \quad(\bmod \alpha(x))
$$

and

(2) there is $x \in L$ such that whenever $a, b \in B$, then

$$
a \approx b \quad(\bmod \beta(x)) \Leftrightarrow \theta(a) \approx \theta(b) \quad(\bmod \pi) .
$$

We will refer to such a function $\theta$ as a $\pi$-demonstrator for $\alpha \rightarrow \beta$.

The next lemma is a consequence of the canonical partition theorem of Prömel and Voigt [6]. This lemma was explicitly stated in Example 3.3 of [8] and was used there to prove the instance (see [8, Corollary 5.5]) of the theorem when $L \cong \Pi(A)$.

Lemma 2. Let $A$ be a finite set and let $\gamma: \Pi(A) \rightarrow \Pi(A)$ be the identity representation. Then for each $m \geq 1$ there is $n$ such that $\gamma^{n} \rightarrow \gamma^{m}$.

The next lemma is the main ingredient in the proof of the theorem.

Lemma 3. Suppose $\alpha: L \rightarrow \Pi(A)$ is a finite congruence representation. Then there is $n \geq 1$ such that $\alpha^{n} \rightarrow \alpha$.

Proof. Let $\alpha: L \rightarrow \Pi(A)$ be a finite congruence representation. Let $(A, \mathscr{F})$ be a unary algebra such that $\alpha: L \rightarrow \Pi(A, \mathscr{F})$ is a lattice isomorphism. Without loss of generality, we assume that the identity function is in $\mathscr{F}($ so $\mathscr{F} \neq \varnothing)$ and that $\mathscr{F}$ is closed under composition. Let $m=|\mathscr{F}| \geq 1$. Letting $\gamma: \Pi(A) \rightarrow$ 
$\Pi(A)$ be the identity function, we can get $n$ by Lemma 2 so that $\gamma^{n} \rightarrow \gamma^{m}$. We claim that $\alpha^{n} \rightarrow \alpha$.

In order to prove that $\alpha^{n} \rightarrow \alpha$, consider some $\pi \in \Pi\left(A^{n}\right)$. To obtain a $\pi$-demonstrator for $\alpha^{n} \rightarrow \alpha$, let $\mathscr{F}=\left\{f_{0}, f_{1}, \ldots, f_{m-1}\right\}$, and let $F: A \rightarrow A^{m}$ be the function for which $F(a)=\left\langle f_{0}(a), f_{1}(a), \ldots, f_{m-1}(a)\right\rangle$ for each $a \in A$. Let $\theta_{1}$ be a $\pi$-demonstrator for $\gamma^{n} \rightarrow \gamma^{m}$, and then let $\theta=\theta_{1} \circ F$. We will show that $\theta$ is a $\pi$-demonstrator for $\alpha^{n} \rightarrow \alpha$. Conditions (1) and (2) in the definition need to be proved.

Proof of (1). Suppose $c, d \in A^{n}$ and $x \in L$. Notice that $\alpha^{k}=\gamma^{k} \circ \alpha$ for $1 \leq k<\omega$. Then each of the following equivalences is clear.

$$
\begin{aligned}
\theta(c) \approx \theta(d)\left(\bmod \alpha^{n}(x)\right) & \Leftrightarrow \theta_{1}(F(c)) \approx \theta_{1}(F(d)) \quad\left(\bmod \gamma^{n}(\alpha(x))\right) \\
& \Leftrightarrow F(c) \approx F(d) \quad\left(\bmod \gamma^{m}(\alpha(x))\right) \\
& \Leftrightarrow F(c) \approx F(d) \quad\left(\bmod \alpha^{m}(x)\right) \\
& \Leftrightarrow \text { for each } i<m, f_{i}(c) \approx f_{i}(d) \quad(\bmod \alpha(x)) \\
& \Leftrightarrow c \approx d \quad(\bmod \alpha(x)) .
\end{aligned}
$$

This proves (1) in the definition.

Proof of (2). Suppose that $\pi \in \Pi\left(A^{n}\right)$, and let $\rho \in \Pi(A)$ be the partition such that

$$
a \approx b \quad(\bmod \rho) \Leftrightarrow \theta(a) \approx \theta(b) \quad(\bmod \pi)
$$

whenever $a, b \in A$. In order to prove (2), we need to show that $\rho \in \Pi(A, \mathscr{F})$. To do this, consider some $i<m$ and arbitrary $a, b \in A$ for which $a \approx b$ $(\bmod \rho)$. Then $\theta(a) \approx \theta(b)(\bmod \pi)$. Hence, there is $\rho_{1} \in \Pi(A, \mathscr{F})$ such that $F(a) \approx F(b)\left(\bmod \gamma^{m}\left(\rho_{1}\right)\right)$, so that for each $k<m, f_{k}(a) \approx f_{k}(b)$ $\left(\bmod \rho_{1}\right)$. Since $\mathscr{F}$ is closed under composition, for each $j<m, f_{j}\left(f_{i}(a)\right) \approx$ $f_{j}\left(f_{i}(b)\right)\left(\bmod \rho_{1}\right)$. It follows that $F\left(f_{i}(a)\right) \approx F\left(f_{i}(b)\right)\left(\bmod \gamma^{m}\left(\rho_{1}\right)\right)$, so that $\theta_{1}\left(F\left(f_{i}(a)\right)\right) \approx \theta_{1}\left(F\left(f_{i}(b)\right)\right)\left(\bmod \gamma^{n}\left(\rho_{1}\right)\right)$; consequently, $\theta\left(f_{i}(a)\right) \approx \theta\left(f_{i}(b)\right)$ $(\bmod \pi)$, so that $f_{i}(a) \approx f_{i}(b)(\bmod \rho)$. This proves $\rho \in \Pi(A, \mathscr{F})$.

The following corollary generalizes Lemma 2.

Corollary 4. Suppose $\alpha: L \rightarrow \Pi(A)$ is a finite congruence representation. Then for each $m \geq 1$ there is $n$ such that $\alpha^{n} \rightarrow \alpha^{m}$.

Proof. This follows immediately from Lemmas 1 and 3 and the observation that $\left(\alpha^{n}\right)^{k}$ and $\alpha^{n k}$ are isomorphic representations.

We can now conclude the following corollary, which involves a technical definition from [8]. The reader is referred to [8] for a precise statement of this definition.

Corollary 5. If $L$ is a finite lattice that has a finite congruence representation, then for each $n<\omega$, L has an $n-C P P$ representation.

Proof. The $n$-CPP representation $\alpha_{n}$ of $L$ is obtained as follows. Let $\alpha$ be a finite congruence representation of $L$. Let $\alpha_{0}=\alpha^{2}$, which by Lemma 1 is a nontrivial finite congruence representation. Recursively obtain finite congruence representations $\alpha_{n+1}$ so that $\alpha_{n+1} \rightarrow \alpha_{n}$. This is possible by Lemmas 1 and 3. The representation $\alpha_{n}$ is an $n$-CPP representation of $L$.

The theorem follows immediately from Corollary 5, Theorem 4.1 of [8], and Remark 5.1 of [8]. 


\section{REFERENCES}

1. W. Feit, An interval in the subgroup lattice of a finite group which is isomorphic to $M_{7}$, Algebra Universalis 17 (1983), 220-221.

2. H. Gaifman, Models and types of Peano's arithmetic, Ann. Math. Logic 9 (1976), 223-306.

3. G. Grätzer and E. T. Schmidt, Characterizations of congruence lattices of abstract algebras, Acta. Sci. Math. (Szeged) 24 (1963), 34-39.

4. J. Paris, On models of arithmetic, Conference in Mathematical Logic, London ' 70 , Lecture Notes in Math., vol. 225, Springer-Verlag, Heidelberg and New York, 1972, pp. 252-280.

5. __ Models of arithmetic and the 1-3-1 lattice, Fund. Math. 95 (1977), 195-199.

6. H. J. Prömel and B. Voigt, Canonical partition theorems for parameter sets, J. Combin. Theory (A) 35 (1983), 309-327.

7. J. H. Schmerl, Extending models of arithmetic, Ann. Math. Logic 14 (1978), 89-109.

8. __ Substructure lattices of models of Peano Arithmetic, Logic Colloquium '84, NorthHolland, Amsterdam, 1986, pp. 225-243.

9. A. Wilkie, On models of arithmetic having non-modular substructure lattices, Fund. Math. 95 (1977), 223-237.

Department of Mathematics, University of Connecticut, Storrs, Connecticut 06269 E-mail address: Schmerl@UCONNVM.UCONN.EDU 